\title{
OBSTRUCTIONS TO A GENERAL CHARACTERIZATION OF GRAPH CORRESPONDENCES
}

\author{
S. KALISZEWSKI ${ }^{\bowtie}$, NURA PATANI and JOHN QUIGG \\ (Received 31 January 2012; accepted 23 January 2013; first published online 7 June 2013) \\ Communicated by G. A. Willis
}

\begin{abstract}
For a countable discrete space $V$, every nondegenerate separable $C^{*}$-correspondence over $c_{0}(V)$ is isomorphic to one coming from a directed graph with vertex set $V$. In this paper we demonstrate why the analogous characterizations fail to hold for higher-rank graphs (where one considers product systems of $C^{*}$-correspondences) and for topological graphs (where $V$ is locally compact Hausdorff), and we discuss the obstructions that arise.
\end{abstract}

2010 Mathematics subject classification: primary 46L08.

Keywords and phrases: Hilbert modules, $C^{*}$-correspondences, $k$-graphs, topological graphs.

\section{Introduction}

Given a directed graph $E$ with countable vertex set $V$, one can construct the graph correspondence $X_{E}$, which is a $C^{*}$-correspondence over $c_{0}(V)$ that encodes the structure of $E$ in a particularly nice way: indeed, we have recently shown in [6] that the assignment $E \mapsto X_{E}$ gives rise to an essentially surjective functor from a category of directed graphs with vertex set $V$ to a category of $c_{0}(V)$-correspondences. In particular, every separable, nondegenerate $c_{0}(V)$-correspondence is isomorphic to a graph correspondence.

In this paper we investigate this issue-characterizing the $C^{*}$-correspondences that come from graphs-in two more general contexts: that of $k$-graphs, which are higherdimensional analogs of directed graphs; and that of topological graphs, which are continuous analogs. It turns out that in each case the most naïve characterization fails: there are $C^{*}$-correspondences that are not isomorphic to any graph correspondence. However, we can in special cases identify obstructions that may lead to a general characterization.

We refer to [11] for the basic definitions and notation regarding directed graphs and their associated $C^{*}$-correspondences, $k$-graphs, and topological $k$-graphs.

(c) 2013 Australian Mathematical Publishing Association Inc. 1446-7887/2013 \$16.00 
In Section 2, we describe product systems associated to $k$-graphs. In particular, we discuss how a $k$-graph may be viewed as a product system over $\mathbb{N}^{k}$ of directed graphs and define an associated product system of $C^{*}$-correspondences. We discuss how each of these product systems may be realized in terms of its skeleton and show how the factorization of paths in the $k$-graph is encoded in the skeleton of the associated product system of correspondences.

In Section 4, we define Hilbert matrices and Hilbert systems and show that Hilbert systems are essentially the same as product systems of $C^{*}$-correspondences. We restrict our attention to the case $k=2$ in Section 5 and establish a characterization of the product systems over $\mathbb{N}^{2}$ of $c_{0}(V)$-correspondences arising from 2-graphs. Further, we specialize to the setting $V=\{v\}$ and identify an isomorphism invariant for product systems over $\mathbb{N}^{2}$ of one-dimensional Hilbert spaces that allows us to identify precisely when such a product system is isomorphic to one associated to a 2-graph.

In Section 6, we turn our attention to topological graphs with vertex space $V$, and initially focus on Hilbert $A$-modules, where $A=C_{0}(V)$. To each Hilbert $A$-module $X$ is associated a Hilbert bundle $p: \mathcal{H} \rightarrow V$. We show that the existence of a continuous choice of orthonormal bases for this Hilbert bundle is equivalent to $X$ being isomorphic to the Hilbert $A$-module constructed from a topological graph using the source map to implement the right $A$-module structure. To incorporate the left $A$-module structure, we consider the representations $\pi_{v}$ coming from the left module action of $A$ on each fiber of the associated Hilbert bundle. For an $A$-correspondence to arise from a topological graph, the continuous choice of orthonormal bases must diagonalize the representation $\pi_{v}$ for each $v \in V$.

To more closely study this, we restrict our attention to the setting where the associated Hilbert bundle has one-dimensional fibers. For such an $A$-correspondence, being isomorphic to a topological graph correspondence is equivalent to the associated Hilbert bundle being trivial. Further specializing to the setting of $A$-imprimitivity bimodules, we identify the Picard invariant of an $A$-imprimitivity bimodule $X$ as the obstruction to $X$ being isomorphic to a topological graph correspondence.

\section{2. $k$-graphs and product systems over $\mathbb{N}^{k}$}

Throughout this section, we consider a fixed countable set $V$. After a brief discussion of product systems, we show how each $k$-graph with vertex set $V$ gives rise to a product system over $\mathbb{N}^{k}$ of $c_{0}(V)$-correspondences. We show that in general, any product system of $c_{0}(V)$-correspondences is determined, up to isomorphism, by a set of basic data we call its skeleton, and we describe the skeleton of the product system of correspondences associated to a $k$-graph in terms of the structure of the $k$-graph.

If $E$ and $F$ are directed graphs with vertex set $V$, the fibered product $E * F$ is the graph with vertex set $V$, edge set

$$
(E * F)^{1}=\left\{(e, f) \in E^{1} \times F^{1} \mid s_{E}(e)=r_{F}(f)\right\},
$$


and range and source maps $r$ and $s$ given by

$$
r(e, f)=r_{E}(e) \quad \text { and } \quad s(e, f)=s_{F}(f) .
$$

If $E^{\prime}$ and $F^{\prime}$ are directed graphs with vertex set $V^{\prime}$, and if $\phi: E \rightarrow E^{\prime}$ and $\psi: F \rightarrow F^{\prime}$ are graph homomorphisms that agree on $V$, then the fibered product homomorphism $\phi * \psi: E * F \rightarrow E^{\prime} * F^{\prime}$ defined on edges by $\phi * \psi(e, f)=(\phi(e), \psi(f))$ and on vertices by $v \mapsto \phi(v)=\psi(v)$ is easily seen to be a graph homomorphism. In particular, if $V^{\prime}=V$ and $\phi, \psi$ are vertex-fixing isomorphisms (that is, $\phi(v)=\psi(v)=v$ ), then $\phi * \psi$ is a graph isomorphism. (This construction makes the set of directed graphs having vertex set $V$ into a tensor groupoid in the language of [5].)

If $S$ is a countable semigroup with identity $e$, then a product system over $S$ of graphs on $V$ is a collection $E=\left\{E_{s} \mid s \in S\right\}$ of directed graphs $E_{s}$ with vertex set $V$ such that $E_{e}=\left(V, V, \mathrm{id}_{V}, \mathrm{id}_{V}\right)$, together with a collection $\alpha=\left\{\alpha_{s, t} \mid s, t \in S\right\}$ of vertexfixing graph isomorphisms $\alpha_{s, t}: E_{s} * E_{t} \rightarrow E_{s t}$ such that $\alpha_{e, s}: E_{e} * E_{s} \rightarrow E_{s}$ and $\alpha_{s, e}$ : $E_{s} * E_{e} \rightarrow E_{s}$ are the natural maps given on edges by $(r(e), e) \mapsto e$ and $(e, s(e)) \mapsto e$ for each $s \in S$, and such that the associativity condition

$$
\alpha_{r s, t} \circ\left(\alpha_{r, s} * \mathrm{id}_{t}\right)=\alpha_{r, s t} \circ\left(\operatorname{id}_{r} * \alpha_{s, t}\right)
$$

holds for all $r, s, t \in S$, where $\mathrm{id}_{t}$ is the identity map on $E_{t}$. (This definition is a special case of [5, Definition 1.1].)

Now let $A$ be a $C^{*}$-algebra. A product system over $S$ of $A$-correspondences is a collection $X=\left\{X_{s} \mid s \in S\right\}$ of $A$-correspondences, together with a collection $\beta=\left\{\beta_{s, t} \mid\right.$ $s, t \in S\}$ of $A$-correspondence isomorphisms $\beta_{s, t}: X_{s} \otimes_{A} X_{t} \rightarrow X_{s t}$ such that $X_{e}=A$ (viewed in the natural way as an $A$-correspondence); $\beta_{e, s}: X_{e} \otimes_{A} X_{s} \rightarrow X_{s}$ and $\beta_{s, e}$ : $X_{s} \otimes_{A} X_{e} \rightarrow X_{s}$ are the natural maps determined by $a \otimes \xi \mapsto a \cdot \xi$ and $\xi \otimes a \mapsto \xi \cdot a$ for each $s \in S$; and the associativity condition $\beta_{r s, t} \circ\left(\beta_{r, s} \otimes \mathrm{id}_{t}\right)=\beta_{r, s t} \circ\left(\mathrm{id}_{r} \otimes \beta_{s, t}\right)$ holds for all $r, s, t \in S$. (Again, see [5, Definition 1.1].)

As observed in [5], product systems over $\mathbb{N}^{k}$ of graphs are essentially the same as $k$-graphs. In more detail, suppose that $(\Lambda, d)$ is a $k$-graph with vertex set $V$ : so $\Lambda$ is a countable category, $d: \Lambda \rightarrow \mathbb{N}^{k}$ is the degree functor, and we have identified the object set $d^{-1}(0)$ with $V$. For each $m \in \mathbb{N}^{k}$, the set $d^{-1}(m)$ is the edge set of a directed graph $E_{m}$ with vertex set $V$ and range and source maps inherited from $\Lambda$. The collection $E=\left\{E_{m} \mid m \in \mathbb{N}^{k}\right\}$ together with the vertex-fixing isomorphisms $\alpha_{m, n}: E_{m} * E_{n} \rightarrow E_{m+n}$ given on edges by

$$
\alpha_{m, n}(\mu, v)=\mu v
$$

is then a product system over $\mathbb{N}^{k}$ of graphs on $V$; moreover, every such product system arises in this way from a $k$-graph with vertex set $V$.

Retaining the above notation, for each $m \in \mathbb{N}^{k}$, let $X_{m}$ be the $c_{0}(V)$-correspondence associated to the graph $E_{m}$; recall (from [11], for example) that by definition

$$
X_{m}=\left\{\xi: E_{m}^{1} \rightarrow \mathbb{C} \mid \text { the map } v \mapsto \sum_{s(e)=v}|\xi(e)|^{2} \text { is in } c_{0}(V)\right\},
$$


with module actions and $c_{0}(V)$-valued inner product given by

$$
(f \cdot \xi \cdot g)(e)=f(r(e)) \xi(e) g(s(e)) \quad \text { and } \quad\langle\xi, \eta\rangle(v)=\sum_{s(e)=v} \overline{\xi(e)} \eta(e) .
$$

Note that $X_{m}$ is densely spanned by the set $\left\{\chi_{e} \mid e \in E_{m}^{1}\right\}$, where $\chi_{e}$ denotes the characteristic function of $\{e\}$. Further, if $p_{v} \in c_{0}(V)$ denotes the characteristic function of a vertex $v \in V$, then for any $e \in E_{m}^{1}$ and $f \in E_{n}^{1}$,

$$
\chi_{e} \otimes \chi_{f}=\chi_{e} \cdot p_{s(e)} \otimes p_{r(f)} \cdot \chi_{f}=\chi_{e} \cdot p_{s(e)} p_{r(f)} \otimes \chi_{f}=0
$$

in $X_{m} \otimes_{c_{0}(V)} X_{n}$ unless $s(e)=r(f)$; thus the balanced tensor product is densely spanned by the set

$$
\left\{\chi_{e} \otimes \chi_{f} \mid(e, f) \in\left(E_{m} * E_{n}\right)^{1}\right\}
$$

For each $m, n \in \mathbb{N}^{k}$ let $\beta_{m, n}: X_{m} \otimes_{c_{0}(V)} X_{n} \rightarrow X_{m+n}$ be the isomorphism determined by

$$
\beta_{m, n}\left(\chi_{e} \otimes \chi_{f}\right)=\chi_{\alpha_{m, n}(e, f)}=\chi_{e f} \quad \text { for }(e, f) \in\left(E_{m} * E_{n}\right)^{1} .
$$

Then $X=\left\{X_{m} \mid m \in \mathbb{N}^{k}\right\}$ with $\beta=\left\{\beta_{m, n} \mid m, n \in \mathbb{N}^{k}\right\}$ is a product system over $\mathbb{N}^{k}$ of $c_{0}(V)$-correspondences (see [12, Proposition 3.2]). We call $(X, \beta)$ the product system of correspondences associated to $(\Lambda, d)$.

Suppose that $(X, \beta)$ is any product system over $\mathbb{N}^{k}$ of $A$-correspondences, and let $\left\{e_{i} \mid 1 \leq i \leq k\right\}$ denote the standard basis for $\mathbb{N}^{k}$. Then setting

$$
Y_{i}=X_{e_{i}} \quad \text { and } \quad T_{i, j}=\beta_{e_{j}, e_{i}}^{-1} \circ \beta_{e_{i}, e_{j}}: Y_{i} \otimes_{A} Y_{j} \rightarrow Y_{j} \otimes_{A} Y_{i}
$$

gives a collection $Y=\left\{Y_{i} \mid 1 \leq i \leq k\right\}$ of $A$-correspondences and a collection $T=\left\{T_{i, j} \mid\right.$ $1 \leq i<j \leq k\}$ of $A$-correspondence isomorphisms such that the hexagonal equation

$$
\left(T_{j, \ell} \otimes \mathrm{id}_{i}\right)\left(\mathrm{id}_{j} \otimes T_{i, \ell}\right)\left(T_{i, j} \otimes \mathrm{id}_{\ell}\right)=\left(\mathrm{id}_{\ell} \otimes T_{i, j}\right)\left(T_{i, \ell} \otimes \mathrm{id}_{j}\right)\left(\mathrm{id}_{i} \otimes T_{j, \ell}\right)
$$

holds for all $1 \leq i<j<\ell \leq k$, where $\mathrm{id}_{i}$ is the identity map on $Y_{i}$. (Note that both sides of (2.2) are isomorphisms $Y_{i} \otimes_{A} Y_{j} \otimes_{A} Y_{\ell} \rightarrow Y_{\ell} \otimes_{A} Y_{j} \otimes_{A} Y_{i}$.) We call the pair $(Y, T)$ the skeleton of $(X, \beta)$.

It follows from [5, Proposition 2.11] (see also Remark 2.1 below) that a product system is uniquely determined, up to isomorphism, by its skeleton. More precisely, if $(Z, \gamma)$ is another product system over $\mathbb{N}^{k}$ of $A$-correspondences, with skeleton $(W, R)$, then $(X, \beta) \cong(Z, \gamma)$ if and only if $(Y, T) \cong(W, R)$ in the sense that there exist isomorphisms $\theta_{i}: Y_{i} \rightarrow W_{i}$ such that the following diagram commutes for each $1 \leq i<$ $j \leq k$.

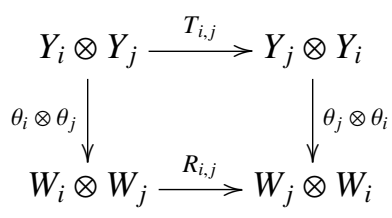


REMARK 2.1. Observe that for $k \leq 2$, there are no hexagonal equations (2.2), and for $k=1$ there are no $T_{i, j}$. Also, defining $T_{j, i}=T_{i, j}^{-1}$ for $i<j$, we have a collection $\left\{T_{i, j} \mid\right.$ $1 \leq i \neq j \leq k\}$ of correspondence isomorphisms that satisfy the hexagonal equations for all distinct $i, j, l$, as in [5]. It is convenient to note that we only need the $T_{i, j}$ for $i<j$.

The following proposition shows how the skeleton encodes the 'commuting squares' (that is, the factorizations of paths of degree $e_{i}+e_{j}$ ) of a $k$-graph.

Proposition 2.2. Suppose that $(\Lambda, d)$ is a k-graph with vertex set $V$, let $(E, \alpha)$ be the associated product system over $\mathbb{N}^{k}$ of graphs, let $(X, \beta)$ be the associated product system of correspondences, and let $(Y, T)$ be the skeleton of $(X, \beta)$. Then for each $1 \leq i<j \leq k$ and each $e \in E_{e_{i}}^{1}$ and $f \in E_{e_{j}}^{1}$,

$$
T_{i, j}\left(\chi_{e} \otimes \chi_{f}\right)=\chi_{\tilde{f}} \otimes \chi_{\tilde{e}}
$$

where $\tilde{f}$ and $\tilde{e}$ are the unique edges in $E_{e_{j}}$ and $E_{e_{i}}$, respectively, such that $\tilde{f} \tilde{e}=e f$ in $E_{e_{i}+e_{j}}$.

Proof. By (2.1), we have $\beta_{e_{i}, e_{j}}\left(\chi_{e} \otimes \chi_{f}\right)=\chi_{e f}$ in $X_{e_{i}+e_{j}}$, and similarly $\beta_{e_{j}, e_{i}}\left(\chi_{\tilde{f}} \otimes \chi_{\tilde{e}}\right)=$ $\chi_{\tilde{f} \tilde{e}}=\chi_{e f}$. Since $T_{i, j}=\beta_{e_{j}, e_{i}}^{-1} \circ \beta_{e_{i}, e_{j}}$ by definition, the result follows.

Since we are interested in determining which product systems come from $k$-graphs, and since the correspondences in the product system associated to a $k$-graph are automatically nondegenerate, we will restrict attention to product systems of nondegenerate correspondences.

\section{Product systems over $\mathbb{N}^{2}$}

To analyze product systems of correspondences over $c_{0}(V)$ in more detail, we need to decompose using the minimal projections $p_{v}:=\chi_{\{v\}}$ for $v \in V$. For arbitrary $k$ this would involve an enormous amount of book-keeping, and would be further complicated by the hexagonal equation (2.2). Therefore we now specialize to the case $k=2$. Some of what we will do (particularly concerning product systems of onedimensional correspondences) can be carried over to the general case.

So, the skeleton $(Y, T)$ of our product system $(X, \beta)$ over $\mathbb{N}^{2}$ has $Y=\left(Y_{1}, Y_{2}\right)$; since we will only be working with two correspondences, we will simplify the notation by letting

$$
\left(Y_{1}, Y_{2}\right)=(Y, Z)
$$

Also, we only have one isomorphism $T_{1,2}$, so we will just write this as $T$. Thus, $T: Y \otimes_{A} Z \stackrel{\cong}{\longrightarrow} Z \otimes_{A} Y$ is an isomorphism of $A$-correspondences, and we denote the skeleton of the product system $(X, \beta)$ by $(Y, Z, T)$.

We use similar notational conventions for isomorphisms between product systems over $\mathbb{N}^{2}$ : if $\left(X^{\prime}, \beta^{\prime}\right)$ is another product system, with skeleton $\left(Y^{\prime}, Z^{\prime}, T^{\prime}\right)$, an 
isomorphism from the first skeleton to the second is an ordered pair $(\theta, \tau)$ of $A$-correspondence isomorphisms

$$
\theta: Y \stackrel{\cong}{\longrightarrow} Y^{\prime} \text { and } \tau: Z \stackrel{\cong}{\longrightarrow} Z^{\prime}
$$

such that the diagram

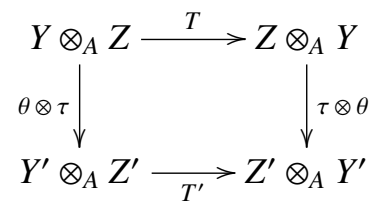

commutes.

\section{Hilbert systems}

We continue to consider a fixed countable set $V$.

Definition 4.1. A Hilbert matrix over $V$ is a family $H=\left\{H_{u v}\right\}_{u, v \in V}$ of Hilbert spaces.

Unless otherwise specified, all our Hilbert matrices will be over $V$.

Definition 4.2. If $H$ and $K$ are Hilbert matrices, an isomorphism of $H$ onto $K$ is a family $S=\left\{S_{u v}\right\}_{u, v \in V}$, where each $S_{u v}$ is a unitary operator from $H_{u v}$ onto $K_{u v}$.

Definition 4.3. The product of two Hilbert matrices $H$ and $K$ is the Hilbert matrix $H * K$, where

$$
(H * K)_{u v}=\bigoplus_{x \in V}\left(H_{u x} \otimes K_{x v}\right) .
$$

Definition 4.4. A Hilbert system over $V$ is a triple $(H, K, S)$, where $H$ and $K$ is are Hilbert matrices and $S: H * K \rightarrow K * H$ is an isomorphism.

Since our Hilbert matrices will be over $V$ by default, so will our Hilbert systems.

Remark 4.5. The existence of an isomorphism $H * K \cong K * H$ for two Hilbert matrices $H$ and $K$ is equivalent to the following relation among the dimensions of the component Hilbert spaces:

$$
\sum_{x \in V}\left(\operatorname{dim} H_{u x}\right)\left(\operatorname{dim} K_{x v}\right)=\sum_{x \in V}\left(\operatorname{dim} K_{u x}\right)\left(\operatorname{dim} H_{x v}\right) \quad \text { for all } u, v \in V .
$$

Definition 4.6. An isomorphism $(\sigma, \tau):(H, K, S) \rightarrow\left(H^{\prime}, K^{\prime}, S^{\prime}\right)$ between Hilbert systems comprises isomorphisms $\sigma: H \rightarrow H^{\prime}$ and $\tau: K \rightarrow K^{\prime}$ making the diagram

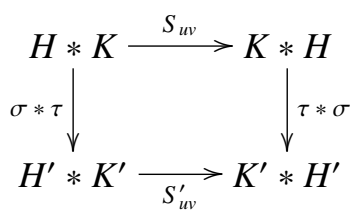


commute, where the fibered product $\sigma * \tau$ has component unitaries

$$
(\sigma * \tau)_{u v}=\bigoplus_{x \in V}\left(\sigma_{u x} \otimes \tau_{x v}\right),
$$

and similarly for $\tau * \sigma$.

We will establish an equivalence between product systems and Hilbert systems. Unsurprisingly, we will work with the skeletons of the product systems. Recall that we put $A=c_{0}(V)$. First we define the Hilbert system associated to a product system.

Definition 4.7. The Hilbert matrix associated to an $A$-correspondence $Y$ is given by $H=\left\{H_{u v}\right\}_{u, v \in V}$ where

$$
H_{u v}=p_{u} \cdot Y \cdot p_{v} .
$$

Here $p_{u}=\chi_{\{u\}}$ is the characteristic function of the singleton set $\{u\}$, regarded as a minimal projection in $A$, and where the one-dimensional ideal $\mathbb{C} p_{v}$ is identified with the complex numbers $\mathbb{C}$.

EXAMPLE 4.8. If $Y=X(E)$ is the graph correspondence associated to a directed graph $E$ with vertex set $V$, then

$$
H_{u v}=\ell^{2}\left(u E^{1} v\right)
$$

Observation 4.9. Let $X$ be a product system over $\mathbb{N}^{2}$ of $A$-correspondences, with skeleton $(Y, Z, T)$, and let $H$ and $K$ be the Hilbert matrices associated to $Y$ and $Z$, respectively, as in Definition 4.7. It follows from the constructions that $H * K$ is the Hilbert matrix associated to the $A$-correspondence $Y \otimes_{A} Z$, and similarly for $K * H$ and $Z \otimes_{A} Y$. Moreover, for each $u, v \in V$ the restriction $\left.T\right|_{(H * K)_{u v}}$ gives a unitary map

$$
S_{u v}:(H * K)_{u v} \rightarrow(K * H)_{u v} .
$$

ExAmple 4.10. In Observation 4.9, if $X$ is the product system of correspondences associated to a 2-graph, with associated product system $\left\{E_{n}\right\}_{n \in \mathbb{N}^{2}}$ of graphs, then

$$
(H * K)_{u v}=\overline{\operatorname{span}}\left\{\chi_{e} \otimes \chi_{f}:(e, f) \in u\left(E_{e_{1}} * E_{e_{2}}\right)^{1} v\right\} .
$$

Moreover,

$$
S_{u v}\left(\chi_{e} \otimes \chi_{f}\right)=\chi_{\tilde{f}} \otimes \chi_{\widetilde{e}},
$$

where $\tilde{f}$ and $\tilde{e}$ are the unique edges in $E_{e_{j}}$ and $E_{e_{i}}$, respectively, such that $\tilde{f} \tilde{e}=e f$ in $E_{e_{i}+e_{j}}$.

Definition 4.11. Let $(Y, Z, T)$ be the skeleton of a product system $X$ over $\mathbb{N}^{2}$ of $A$-correspondences. The Hilbert system associated to $X$ is $(H, K, S)$, where $S=\left\{S_{u v}\right\}$ is the family of unitaries defined in Observation 4.9.

We now turn to the task of defining the product system associated to a Hilbert system. 
Observation 4.12. Let $H$ be a Hilbert matrix over $V$. Then for every $u, v \in V$ we can define an $A$-correspondence structure on $H_{u v}$ as follows. For $\xi, \eta \in H_{u v}$ and $f \in A$ we let:

- $\quad \xi \cdot f=\xi f(v)$

- $f \cdot \xi=f(u) \xi$

- $\langle\xi, \eta\rangle_{A}=\langle\xi, \eta\rangle p_{v}$

where on the right-hand side of the last equation the unadorned inner product $\langle\xi, \eta\rangle$ is the one given by the Hilbert space $H_{u v}$. Note that in order to facilitate the connection with correspondences we adopt the physicists' convention that the Hilbert space inner product is linear in the second variable!

Definition 4.13. Let $H$ be a Hilbert matrix over $V$, and regard the Hilbert spaces $H_{u v}$ as $A$-correspondences as in Observation 4.12. Then the A-correspondence associated to $H$ is

$$
Y:=\bigoplus_{u, v \in V} H_{u v},
$$

regarded as a direct sum of $H$-correspondences.

Observation 4.14. Let $H$ and $K$ be Hilbert matrices over $V$, and let $Y$ and $Z$ be the $A$-correspondences associated to $H$ and $K$, respectively, as in Definition 4.13. Further let $S: H \rightarrow K$ be a Hilbert-matrix isomorphism. Then each $S_{u v}: H_{u v} \rightarrow K_{u v}$ can be regarded as an $A$-correspondence isomorphism, and the direct sum

$$
T=\bigoplus_{u, v \in V} S_{u v}: Y \rightarrow Z
$$

is also an $A$-correspondence isomorphism.

Definition 4.15. With the notation of Observation 4.14, we call $T: Y \rightarrow Z$ the A-correspondence isomorphism associated to $S$.

Definition 4.16. Let $(H, K, S)$ be a Hilbert system, and let $Y$ and $Z$ be the $A$-correspondences associated to $H$ and $K$, respectively, as in Definition 4.13. Then the product system associated to $(H, K, S)$ is the unique product system determined by the skeleton $(Y, Z, T)$, where $T: Y \otimes_{A} Z \rightarrow Z \otimes_{A} Y$ is the $A$-correspondence isomorphism associated to the Hilbert-matrix isomorphism $S: H * K \rightarrow K * H$ as in Definition 4.15.

THeOREM 4.17.

(i) Let $X$ be a product system over $\mathbb{N}^{2}$ of A-correspondences, let $(H, K, S)$ be the associated Hilbert system, and let $X^{\prime}$ be the product system associated to $(H, K, S)$. Then $X \cong X^{\prime}$.

(ii) Let $(H, K, S)$ be a Hilbert system, let $X$ be the associated product system, and let $\left(H^{\prime}, K^{\prime}, S^{\prime}\right)$ be the Hilbert system associated to $X$. Then $(H, K, S) \cong\left(H^{\prime}, K^{\prime}, S^{\prime}\right)$.

Proof. (i) If $(Y, Z, T)$ and $\left(Y^{\prime}, Z^{\prime}, T^{\prime}\right)$ are the corresponding skeletons of $X$ and $X^{\prime}$, respectively, then $Y$ is the internal direct sum of the $A$-correspondences $H_{u v}$, while $Y^{\prime}$ is their external direct sum, and similarly for $Z, Z^{\prime}$, and the $K_{u v}$. The canonical 
isomorphism between internal and external direct sums is easily seen to give an isomorphism $X \cong X^{\prime}$.

(ii) If $(Y, Z, T)$ is the skeleton of $X$, then $Y$ is the external direct sum of the $H_{u v}$, while each $H_{u v}$ is a sub-correspondence of $Y^{\prime}$, and similarly for $Z, K_{u v}$, and $K_{u v}^{\prime}$. It is easy to check that if we take, for every $u, v \in V, \sigma_{u v}$ to be the canonical inclusion of $H_{u v}$ into $Y$, and similarly for $\tau_{u v}$ including $K_{u v}$ into $Z$, then $(\sigma, \tau):(H, K, S) \rightarrow\left(H^{\prime}, K^{\prime}, S^{\prime}\right)$ is an isomorphism.

REMARK 4.18. Theorem 4.17 says that the passage between product systems and Hilbert systems is an inverse process up to isomorphism. In fact, this could be promoted to an equivalence of categories, but since we have no applications in mind we will not make this precise.

\section{The obstruction for 2-graphs}

For the case $k=2$, our characterization problem becomes the following.

Question 5.1. Which product systems are isomorphic to product systems of correspondences associated to 2-graphs?

The fact that product systems over $\mathbb{N}^{2}$ of $A$-correspondences are completely characterized by their associated Hilbert systems will lead us to one answer to the above question.

Definition 5.2. Let $H$ and $K$ be Hilbert matrices. For $u, v \in V$ let $\mathcal{E}_{u, v}$ and $\mathcal{F}_{u, v}$ be orthonormal bases for the Hilbert spaces $H_{u, v}$ and $K_{u, v}$, respectively. Put

$$
(\mathcal{E} * \mathcal{F})_{u, v}=\left\{e \otimes f: e \in \mathcal{E}_{u, x}, f \in \mathcal{F}_{x, v} \text { for some } x \in V\right\} .
$$

ObServation 5.3. With the above notation, $(\mathcal{E} * \mathcal{F})_{u, v}$ is an orthonormal basis of $(H * K)_{u, v}$.

THeOREM 5.4. Let $X$ be a product system over $\mathbb{N}^{2}$ of $c_{0}(V)$-correspondences, and let $(H, K, S)$ be the associated Hilbert system. Then $X$ is isomorphic to the product system of correspondences associated to a 2-graph with vertex set $V$ if and only if there are choices $\mathcal{E}_{u, v}$ and $\mathcal{F}_{u, v}$ of orthonormal bases for the $H_{u, v}$ and $K_{u, v}$, respectively, such that for all $u, v \in V$,

$$
S_{u, v}\left((\mathcal{E} * \mathcal{F})_{u, v}\right)=(\mathcal{F} * \mathcal{E})_{u, v} .
$$

PROof. It is routine to verify that the stated property is an isomorphism invariant of Hilbert systems, and Example 4.10 shows that it is satisfied when $X$ is the product system of correspondences associated to a 2-graph.

REMARK 5.5. Relaxing the criterion in Theorem 5.4 a little bit, we can make contact with the $k$-graph cohomology of [8], although we only pursue it in the case $k=2$. Recall that on a 2-graph $\Lambda$ an element of $Z^{2}(\Lambda, \mathbb{T})$ is just a function $\phi: \Lambda^{(1,1)} \rightarrow$ $\mathbb{T}$, and $\phi, \phi^{\prime} \in Z^{2}(\Lambda, \mathbb{T})$ are cohomologous if there exists $\alpha \in Z^{1}(\Lambda, \mathbb{T})$, that is, a 
function $\alpha: \Lambda^{(1,0)} \cup \Lambda^{(0,1)} \rightarrow \mathbb{T}$ satisfying $\alpha(e) \alpha(f)=\alpha\left(f^{\prime}\right) \alpha\left(e^{\prime}\right)$ whenever $e f$ and $f^{\prime} e^{\prime}$ are the two edge-path factorizations of an element of $\Lambda^{(1,1)}$, such that

$$
\alpha(e) \alpha(f) \phi(e f)=\alpha\left(f^{\prime}\right) \alpha\left(e^{\prime}\right) \phi^{\prime}\left(f^{\prime} e^{\prime}\right)
$$

in all such cases.

Consider the following relaxation of the criterion in Theorem 5.4. Suppose that there are choices $\mathcal{E}_{u, v}$ and $\mathcal{F}_{u, v}$ of orthonormal bases for $H_{u, v}$ and $K_{u, v}$, respectively, such that for all $u, v \in V$ and $(e, f) \in(\mathcal{E} * \mathcal{F})_{u v}$ there exists $\left(f^{\prime}, e^{\prime}\right) \in(\mathcal{F} * \mathcal{E})_{u v}$ such that $S_{u v}(e \otimes f)$ is a scalar multiple of $f^{\prime} \otimes e^{\prime}$. Equivalently, suppose that we have a bijection $\theta: \mathcal{E} * \mathcal{F} \rightarrow \mathcal{F} * \mathcal{E}$ and a map $\phi_{0}: \mathcal{E} * \mathcal{F} \rightarrow \mathbb{T}$ such that if $\left(f^{\prime}, e^{\prime}\right)=\theta(e, f)$ then

$$
S_{u v}(e \otimes f)=\phi_{0}(e, f)\left(f^{\prime} \otimes e^{\prime}\right) .
$$

By the discussion in [7, Section 6], there is a unique 2-graph $\Lambda$, with $\Lambda^{(1,0)}=\mathcal{E}$ and $\Lambda^{(0,1)}=\mathcal{F}$, such that $\theta(e, f)=\left(f^{\prime}, e^{\prime}\right)$ in $\mathcal{F} * \mathcal{E}$ if and only if $e f=f^{\prime} e^{\prime}$ in $\Lambda^{(1,1)}$. It then follows that there is a unique $\phi \in Z^{2}(\Lambda, \mathbb{T})$ such that for all $\lambda \in \Lambda^{(1,1)}$, if $\lambda=e f$ for $(e, f) \in \mathcal{E} * \mathcal{F}$ then

$$
\phi(\lambda)=\phi_{0}(e, f) .
$$

We can recover the Hilbert system $(Y, Z, S)$, and hence the product system $X$, up to isomorphism, from $\Lambda$ and $\phi$ : for example, we can take $Y$ to be the completion of the free vector space generated by $\mathcal{E}$, completed with respect to the unique inner product in which $\mathcal{E}$ is orthonormal, and similarly for $Z$ and $\mathcal{F}$, and then the cocycle $\phi$ clearly determines the unitaries $S_{u v}$.

The Cuntz-Pimsner algebra $O_{X}$ is isomorphic to the twisted 2-graph algebra $C_{\phi}^{*}(\Lambda)$ of [8]. By [8, Proposition 7.6], if $\phi$ is homologous to $\phi^{\prime}$ then $C_{\phi}^{*}(\Lambda) \cong C_{\phi^{\prime}}^{*}(\Lambda)$. In fact, a little more is true: the associated product systems are isomorphic. To see this, suppose that $\phi$ and $\phi^{\prime}$ are related as in (5.1), and let $X$ and $X^{\prime}$ be the associated product systems, with associated Hilbert systems $(H, K, S)$ and $\left(H, K, S^{\prime}\right)$, respectively-to be clear, we build the Hilbert matrices $H$ and $K$ such that, for every $u, v \in V, u\left(\Lambda^{(1,0)}\right) v$ and $u\left(\Lambda^{(0,1)}\right) v$ are orthonormal bases for $H_{u v}$ and $K_{u v}$, respectively; the only difference between $X$ and $X^{\prime}$ is that the isomorphism $S: H * K \rightarrow K * H$ is built using $\phi$ and $S^{\prime}: H * K \rightarrow K * H$ is built using $\phi^{\prime}$. For each $u, v \in V$ let $\sigma_{u v}: H_{u v} \rightarrow H_{u v}^{\prime}$ be the unique unitary such that

$$
\sigma_{u v} e=\alpha(e) e \text { for } e \in \mathcal{E},
$$

and similarly for $\tau_{u v}: K_{u v} \rightarrow K_{u v}^{\prime}$. It is routine to check that $(\sigma, \tau):(H, K, S) \rightarrow$ $\left(H, K, S^{\prime}\right)$ is an isomorphism.

Product systems of Hilbert spaces. Theorem 5.4 does not seem to give a very practical test. The main difficulty in applying it would lie in analyzing the unitaries

$$
S_{u v}: \bigoplus_{x \in V}\left(H_{u x} \otimes K_{x v}\right) \rightarrow \bigoplus_{x \in V}\left(K_{u x} \otimes H_{x v}\right) ;
$$

$S_{u v}$ need not have any relation to the direct-sum decompositions. 
In order to obtain a more practical test, we specialize to the case of one vertex, that is, $V=\{v\}$. Then we can regard the Hilbert system $(H, K, S)$ as comprising a single unitary $S$ between Hilbert spaces $H$ and $K$, and $X$ becomes a product system of Hilbert spaces. Moreover, if $X^{\prime}$ is another such product system of Hilbert spaces, with associated Hilbert system $\left(H^{\prime}, K^{\prime}, S^{\prime}\right)$, then $X \cong X^{\prime}$ if and only if there are unitaries $\sigma: H \rightarrow H^{\prime}$ and $\tau: K \rightarrow K^{\prime}$ making the diagram

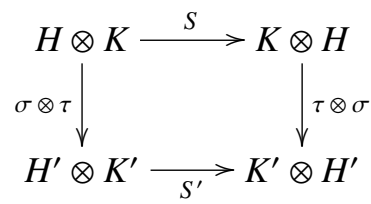

commute.

We will get a more manageable invariant by composing with the flip: let $\Sigma$ : $H \otimes K \rightarrow K \otimes H$ be the flip map, and define

$$
\omega=\Sigma S,
$$

which is a unitary operator on the Hilbert space $H \otimes K$.

Definition 5.6. With the above notation, we call $\omega$ the flipped unitary associated to the product system $X$ over $\mathbb{N}^{2}$ of Hilbert spaces.

Definition 5.7. Let $H$ and $K$ be Hilbert spaces. Let us say that two unitary operators $\omega$ and $\omega^{\prime}$ on $H \otimes K$ are tensor equivalent if there exist unitaries $\sigma \in \mathcal{L}(H)$ and $\tau \in \mathcal{L}(K)$ such that

$$
\omega^{\prime}=\operatorname{Ad}(\sigma \otimes \tau) \omega
$$

The class of the flipped unitary under tensor equivalence gives a complete isomorphism invariant for product systems over $\mathbb{N}^{2}$ of Hilbert spaces. If we say that a unitary operator on a Hilbert space is a permutation unitary if it can be represented as a permutation matrix with respect to some orthonormal basis, then the criterion of Theorem 5.4 becomes the following corollary.

Corollary 5.8. A product system $X$ over $\mathbb{N}^{2}$ of Hilbert spaces is isomorphic to the product system of correspondences associated to a 2-graph $\Lambda$ with a single vertex if and only if its flipped unitary is tensor equivalent in the sense of Definition 5.7 to an elementary tensor $U_{H} \otimes U_{K}$ of permutation unitaries on $H$ and $K$.

The above results take the following particularly simple form for a single vertex and one-dimensional Hilbert spaces.

Corollary 5.9. Let $X$ be a product system over $\mathbb{N}^{2}$ of one-dimensional Hilbert spaces, with flipped unitary $\omega$. Then $\omega$ may be identified with an element of the unit circle $\mathbb{T}$, and the isomorphism classes of such product systems are parameterized by $\mathbb{T}$. Moreover, $X$ is isomorphic to the product system of correspondences associated to a 2-graph if and only if $\omega=1$. 
Proof. Let $(H, K, S)$ be the Hilbert system associated to $X$. Since $H$ and $K$ are onedimensional, so is $H \otimes K$, so the unitary operator $\omega$ is a scalar multiple of the identity map on a one-dimensional Hilbert space, and this scalar must be an element of $\mathbb{T}$. In fact, $\omega$ can be identified with this element of $\mathbb{T}$, and since any unitary operator on $H \otimes K$ can occur as a flipped unitary of a suitable $X$, any element of $\mathbb{T}$ can arise in this way. It is obvious that tensor equivalence on the set of unitary operators on the one-dimensional Hilbert space $H \otimes K$ is just equality. For the last statement, just observe that an elementary tensor of permutation unitaries on the one-dimensional Hilbert spaces $H$ and $K$ is associated to the element 1 of $\mathbb{T}$.

REMARK 5.10. Thus, even in the trivial case of one-dimensional correspondences over $\mathbb{C}$, there are uncountably many product systems over $\mathbb{N}^{2}$ that are not isomorphic to product systems of correspondences associated to 2-graphs.

It is interesting to identify the $C^{*}$-algebra $O_{X}$ of a product system over $\mathbb{N}^{2}$ of onedimensional Hilbert spaces $H$ and $K$ : let $\omega$ be the flipped unitary, which we identify with an element of $\mathbb{T}$ as in Corollary 5.9. Choosing unit vectors $e$ and $f$ in $H$ and $K$, respectively, it is easy to see using induction that Cuntz-Pimsner covariant representations of the product system $X$ correspond to an assignment $e \mapsto u$ and $f \mapsto v$ of unitaries satisfying the relation $u v=\omega v u$, and so, letting $\omega=e^{i \theta}$, it follows that $O_{X}$ is isomorphic to the rotation algebra with angle $\theta$ [9, Proposition 5.1.5].

REMARK 5.11. It follows from [5] that the skeleton of a product system $(X, \beta)$ over $\mathbb{N}$ of $c_{0}(V)$-correspondences is just a single correspondence $Y$; then by [6, Theorem 1.1] we have $Y \cong X_{E}$ for some directed graph $E$, and thus we quickly conclude that $(X, \beta)$ is isomorphic to the product system of correspondences associated to a 1-graph. More generally, every product system $(X, \beta)$ over a finitely-generated free semigroup $S$ of $c_{0}(V)$-correspondences is isomorphic to one associated to a product system over $S$ of graphs. To see this, note that, in the terminology of Fowler and Sims [5], $S$ is a rightangled Artin semigroup with no commutation relations, so the skeleton $Y$ of $(X, \beta)$ has no $T_{i, j}$; again by [6] each $Y_{i}$ is isomorphic to a graph correspondence $X_{E_{i}}$, and the $E_{i}$ form the skeleton of a product system over $S$ of graphs, whose associated product system of correspondences can be checked to be isomorphic to $(X, \beta)$ by examining the skeletons. Thus when the semigroup $S$ is free and finitely-generated there is no obstruction to a product system over $S$ of $c_{0}(V)$-correspondences coming from a product system over $S$ of graphs.

\section{Topological graphs}

Now let $V$ be a fixed locally compact Hausdorff space, and let $A=C_{0}(V)$. For any topological graph $E=\left(E^{1}, V, r, s\right)$ with vertex space $V$, let $X(E)$ denote the associated $A$-correspondence. In this section our characterization problem becomes the following.

Question 6.1. Which $A$-correspondences are isomorphic to $X(E)$ for some topological graph with vertex space $V$ ? 
This question can be fruitfully separated into two parts.

Questions 6.2.

(1) Which Hilbert $A$-modules are isomorphic to $X\left(E^{1}, s\right)$ for some local homeomorphism $s: E^{1} \rightarrow V$ ? Here we use self-explanatory notation: if $E$ is a topological graph, then forgetting the range map $r: E^{1} \rightarrow V$, and forgetting the left $A$-module structure, the $A$-correspondence $X(E)$ becomes a Hilbert $A$ module $X\left(E^{1}, s\right)$ that only depends upon the source map $s$, and can be formed from any locally compact Hausdorff space $E^{1}$ and any local homeomorphism $s: E^{1} \rightarrow V$.

(2) For Hilbert $A$-modules of the form $X=X\left(E^{1}, s\right)$, which left $A$-module structures make $X$ into a topological graph correspondence $X(E)$ for a suitable range map $r$ ?

We need to use the correspondence between Hilbert $A$-modules and continuous Hilbert bundles (for the case of compact $V$, see, for example, [14, 15]; the references $[3,4]$ are also useful), which we briefly recall here in simplified form. First, if $p: \mathcal{H} \rightarrow V$ is a continuous Hilbert bundle, then the space $\Gamma_{0}(\mathcal{H})$ of $C_{0}$-sections becomes a Hilbert $A$-module with operations

$$
\begin{gathered}
\langle\xi, \eta\rangle_{A}(v)=\langle\xi(v), \eta(v)\rangle, \\
(\xi \cdot f)(v)=\xi(v) f(v),
\end{gathered}
$$

for $\xi, \eta \in \Gamma_{0}(\mathcal{H})$ and $f \in A$. In the other direction, let $X$ be a Hilbert $A$-module. For each $v \in V$, define a positive semidefinite Hermitian form $\langle\cdot, \cdot\rangle_{v}$ on $X$ by

$$
\langle\xi, \eta\rangle_{v}=\langle\xi, \eta\rangle_{A}(v) \text {. }
$$

Then the Hausdorff completion of $X$ with respect to $\langle\cdot, \cdot\rangle_{v}$ is a Hilbert space $H_{v}$. Let $Q_{v}: X \rightarrow H_{v}$ be the associated map. Let $\mathcal{H}=\bigsqcup_{v \in V} H_{v}$, and define $\Phi: X \rightarrow \prod_{v \in V} H_{v}$ by

$$
(\Phi \xi)(v)=Q_{v} \xi
$$

Then there is a unique topology on $\mathcal{H}$ making it into a continuous Hilbert bundle over $V$ such that $\Phi$ is an isometric isomorphism of $X$ onto $\Gamma_{0}(\mathcal{H})$, and then in fact $\Phi$ is a Hilbert $A$-module isomorphism. Letting $J_{v}=\{f \in A: f(v)=0\}$ be the associated maximal ideal, we can equivalently identify

$$
H_{v}=X /\left(X \cdot J_{v}\right),
$$

which, by the general theory of Hilbert modules, is naturally a Hilbert $A / J_{v}$-module, and then $Q_{v}: X \rightarrow H_{v}$ is just the quotient map.

Now consider the case $X=X\left(E^{1}, s\right)$. Here we can take

$$
H_{v}=\ell^{2}\left(s^{-1}(v)\right)
$$

and then $Q_{v}: X \rightarrow H_{v}$ is given by $Q_{v} \xi=\left.\xi\right|_{s^{-1}(v)}$, and $\Phi$ is given by

$$
(\Phi \xi)(v)=\sum_{s(e)=v} \xi(e) \chi_{e},
$$

where we write $\chi_{e}$ for the characteristic function of the singleton $\{e\}$. 
We will give one answer to Question 6.2(1) using the following concept.

Definition 6.3. Let $p: \mathcal{H} \rightarrow V$ be a continuous Hilbert bundle. We say that a subset $\mathcal{E} \subset \mathcal{H}$ is a continuous choice of orthonormal bases for $\mathcal{H}$ if:

(i) for each $v \in V$, the intersection $\mathcal{E}_{v}:=\mathcal{E} \cap H_{v}$ is an orthonormal basis for the Hilbert space $H_{v}$;

(ii) the restriction of the bundle projection $p$ to $\mathcal{E}$ is a local homeomorphism.

Theorem 6.4. A Hilbert A-module $X$ is isomorphic to $X\left(E^{1}, s\right)$ for some local homeomorphism $s: E^{1} \rightarrow V$ if and only if the associated Hilbert bundle $p: \mathcal{H} \rightarrow V$ has a continuous choice of orthonormal bases.

Proof. First assume that $X=X\left(E^{1}, s\right)$ for a local homeomorphism $s: E^{1} \rightarrow V$. Define $\gamma: E^{1} \rightarrow \mathcal{H}$ by

$$
\gamma(e)=\chi_{e},
$$

and let $\mathcal{E}=\gamma\left(E^{1}\right)$.

Claim. $\gamma$ is a homeomorphism of $E^{1}$ onto $\mathcal{E}$. To see this, note first of all that $\gamma$ is certainly injective. To see that $\gamma$ is continuous, let $e_{0} \in E^{1}$, and choose a neighborhood $U$ of $e_{0}$ on which $s$ restricts to a homeomorphism. Then choose $\xi \in C_{c}\left(E^{1}\right)$ such that $\operatorname{supp} \xi \subset U$ and $\xi$ is identically 1 on a smaller neighborhood $W$ of $e$. Then

$$
(\Phi \xi)(s(e))=\xi(e) \chi_{e} \quad \text { for all } e \in U,
$$

so for all $e \in W$,

$$
\gamma(e)=\chi_{e}=(\Phi \xi)(s(e))
$$

which is continuous in $e$ because $\Phi \xi$ is a continuous section of $\mathcal{H}$.

To see that $\gamma$ is open onto its image, let $N$ be an open neighborhood of $e_{0}$ in $E^{1}$ such that $s$ maps $N$ homeomorphically onto an open set in $V$. Put $W=\gamma(N)$. Then on $W$ the inverse of $\gamma$ is given by

$$
\gamma^{-1}=\tau \circ p
$$

where $\tau: s(N) \rightarrow N$ is the inverse of the homeomorphism

$$
\left.s\right|_{N}: N \rightarrow s(N)
$$

and $p: \mathcal{H} \rightarrow V$ is the bundle projection. Since both $p$ and $\tau$ are continuous, we see that

$$
W=\left(\gamma^{-1}\right)^{-1}(N)=p^{-1}\left(\tau^{-1}(N)\right) \cap \gamma\left(E^{1}\right)
$$

is open, and the claim is verified.

For each $v \in V$,

$$
\mathcal{E}_{v}:=\mathcal{E} \cap H_{v}=\left\{\chi_{e}\right\}_{e \in s^{-1}(v)}
$$


is an orthonormal basis for the fiber $H_{v}=\ell^{1}\left(s^{-1}(v)\right)$ of $\mathcal{H}$. The commutative diagram

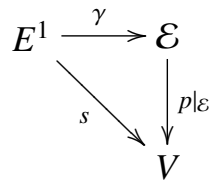

shows that $\left.p\right|_{\mathcal{E}}$ is a local homeomorphism because $\gamma$ is a homeomorphism.

Conversely, suppose that we have a continuous choice $\mathcal{E}$ of orthonormal bases for $\mathcal{H}$. Let $s=\left.p\right|_{\mathcal{E}}$, which is a local homeomorphism by hypothesis. We will construct an isomorphism $\Psi: X(\mathcal{E}, s) \rightarrow X$ of Hilbert $A$-modules. For $\xi \in X(\mathcal{E}, s)$ define a section $\Psi \xi$ of the Hilbert bundle $\mathcal{H}$ by

$$
(\Psi \xi)(v)=\sum_{e \in \mathcal{E}_{v}} \xi(e) e,
$$

where, as above, $\mathcal{E}_{v}=\mathcal{E} \cap H_{v}$ is an orthonormal basis of the fiber $H_{v}$. Note that $\mathcal{E}_{v}=s^{-1}(v)$. Then

$$
\|(\Psi \xi)(v)\|^{2}=\sum_{e \in s^{-1}(v)}|\xi(e)|^{2}=\langle\xi, \xi\rangle_{C_{0}(V)}(v),
$$

so $\Psi \xi \in \Gamma_{0}(\mathcal{H})$. Clearly $\Psi: X(\mathcal{E}, s) \rightarrow \Gamma_{0}(\mathcal{H})$ is linear. We show that it preserves inner products: for $\xi, \eta \in X(\mathcal{E}, s)$ and $v \in V$,

$$
\begin{aligned}
\langle\Psi \xi, \Psi \eta\rangle_{C_{0}(V)}(v) & =\langle(\Psi \xi)(v),(\Psi \eta)(v)\rangle \\
& =\sum_{e \in s^{-1}(v)}\langle\xi(e) e, \eta(e) e\rangle \\
& =\sum_{e \in s^{-1}(v)} \overline{\xi(e)} \eta(e) \\
& =\langle\xi, \eta\rangle_{C_{0}(V)}(v) .
\end{aligned}
$$

It follows that $\Psi$ preserves the right $A$-module structures. It now remains to show that $\Psi$ is surjective, and for this it suffices to show that the set $\Psi(X(\mathcal{E}, s))$ of sections is a $C_{0}(V)$-submodule that is total in each fiber: the first holds since $\Psi$ preserves the right $A$-module structures, and for the second we see that, for each $v \in V$,

$$
\{(\Psi \xi)(v): \xi \in X(\mathcal{E}, s)\} \quad \text { is total in } H_{v},
$$

because it contains $\mathcal{E}_{v}$, since we can take $\xi \in X(\mathcal{E}, s)$ that is 1 at any given $e \in \mathcal{E}_{v}$ and is 0 at all other $e^{\prime} \in s^{-1}(v)$.

Now we incorporate the left $A$-module structure. Given an $A$-correspondence $X$, we know from the above that, at least as far as the Hilbert $A$-module structure goes, we can assume that $X=\Gamma_{0}(\mathcal{H})$ for a continuous Hilbert bundle $p: \mathcal{H} \rightarrow V$, and then for 
each $v \in V$, since $\phi(A)$ acts on $X$ by right $A$-module maps, there is a representation $\pi_{v}$ of $A$ on the fiber $H_{v}$ such that

$$
(\phi(f) \xi)(v)=\pi_{v}(f) \xi(v) \quad \text { for } \xi \in X .
$$

Corollary 6.5. Let $p: \mathcal{H} \rightarrow V$ be a continuous Hilbert bundle, let $X=\Gamma_{0}(\mathcal{H})$ be the associated Hilbert A-module, and suppose that $\phi: A \rightarrow \mathcal{L}(X)$ is a nondegenerate homomorphism. Then the A-correspondence $X$ is isomorphic to $X(E)$ for some topological graph $E$ if and only if $\mathcal{H}$ has a continuous choice $\mathcal{E}$ of orthonormal bases such that for each $v \in V, \mathcal{E}_{v}=\mathcal{E} \cap H_{v}$ diagonalizes the representation $\pi_{v}$ of $A$ on $H_{v}$ associated to the left module action $\phi: A \rightarrow \mathcal{L}(X)$.

Proof. First assume that $X=X(E)$ for a topological graph $E=\left(E^{1}, V, r, s\right)$. Then, as in Theorem 6.4, we can take $\mathcal{E}=\gamma\left(E^{1}\right)$ as our continuous choice of orthonormal bases. For each $f \in A, v \in V$, and $e \in \mathcal{E}_{v}$,

$$
\pi_{v}(f) \gamma(e)=\pi_{v}(f) \chi_{e}=f(r(e)) \chi_{e}
$$

so $\gamma(e)$ is an eigenvector of $\pi_{v}(f)$. Thus the orthonormal basis $\mathcal{E}_{v}$ of $H_{v}$ diagonalizes the representation $\pi_{v}$.

Conversely, suppose that we have a continuous choice $\mathcal{E}$ of orthonormal bases for $\mathcal{H}$ such that $\mathcal{E}_{v}$ diagonalizes $\pi_{v}$ for every $v \in V$. By Theorem 6.4 we may assume that $X=X\left(E^{1}, s\right)$ for a local homeomorphism $s: E^{1} \rightarrow V$, and that $\mathcal{E}=\left\{\chi_{e}: e \in E^{1}\right\}$. Our hypothesis then says that for each $e \in E^{1}, \chi_{e}$ is a common eigenvector for the operators $\left\{\pi_{v}(f): f \in A\right\}$, and hence by restriction we have a one-dimensional representation of $A$ on the subspace $\mathbb{C} \chi_{e}$; therefore there is a unique $r(e) \in V$ such that

$$
\pi_{v}(f) \chi_{e}=f(r(e)) \chi_{e} \quad \text { for } f \in A \text {. }
$$

We must show two things:

(1) $r: E^{1} \rightarrow V$ is continuous, so that $E:=\left(E^{1}, V, r, s\right)$ is a topological graph; and

(2) for $f \in A, \xi \in X$, and $e \in E^{1}$,

$$
(\phi(f) \xi)(e)=f(r(e)) \xi(e)
$$

For (1), let $e_{0} \in E^{1}$, and choose $\xi \in C_{c}\left(E^{1}\right) \subset X$ that is identically 1 on a neighborhood $U$ of $e_{0}$. It suffices to show that for all $f \in A$ the function $e \mapsto f(r(e))$ is continuous at $e_{0}$. But for $e \in U$,

$$
f(r(e))=f(r(e)) \xi(e)=(\phi(f) \xi)(e),
$$

and the function $\phi(f) \xi$ is continuous. 
For (2), let $v=s(e)$, and note that

$$
\begin{aligned}
\left.(\phi(f) \xi)\right|_{s^{-1}(v)} & =\left.\pi_{v}(f) \xi\right|_{s^{-1}(v)} \\
& =\pi_{v}(f) \sum_{e \in s^{-1}(v)} \xi(e) \chi_{e} \\
& =\sum_{e \in s^{-1}(v)} \xi(e) \pi_{v}(f) \chi_{e} \\
& =\sum_{e \in s^{-1}(v)} \xi(e) f(r(e)) \chi_{e}
\end{aligned}
$$

and that

$$
\left.(\phi(f) \xi)\right|_{s^{-1}(v)}=\left.\pi_{v}(f) \xi\right|_{s^{-1}(v)},
$$

so that

$$
(\phi(f) \xi)(e)=\sum_{e^{\prime} \in s^{-1}(v)} \xi\left(e^{\prime}\right) f\left(r\left(e^{\prime}\right)\right) \chi_{e}^{\prime}(e)=f(r(e)) \xi(e) .
$$

In order to introduce our obstruction to an $A$-correspondence coming from a topological graph, we specialize to the case of one-dimensional fibers. As above, we separate into two steps, starting with Hilbert modules.

Corollary 6.6. Let $X$ be a Hilbert A-module whose associated Hilbert bundle $p$ : $\mathcal{H} \rightarrow V$ has one-dimensional fibers. Then $X$ is isomorphic to $X\left(E^{1}, s\right)$ for some local homeomorphism $s: E^{1} \rightarrow V$ if and only if $\mathcal{H}$ is trivial.

Proof. First assume that $\mathcal{H}$ is trivial. Then after applying a Hilbert bundle isomorphism (equivalently, a Hilbert module isomorphism), we may assume that $\mathcal{H}$ is a product bundle $H \times V$, with bundle projection equal to the coordinate projection onto the second factor. Let $B$ be an orthonormal basis of the Hilbert space $H$. Then it is routine to verify that $\mathcal{E}:=B \times V$ is a continuous choice of orthonormal bases for the product bundle. Thus $X \cong X\left(E^{1}, s\right)$ for some local homeomorphism $s: E^{1} \rightarrow V$.

For the converse, it suffices to show that if $X=X\left(E^{1}, s\right)$ for some local homeomorphism, and if the associated Hilbert bundle $p: \mathcal{H} \rightarrow V$ has one-dimensional fibers, then $\mathcal{H}$ is trivial. Define $\gamma: E^{1} \rightarrow \mathcal{H}$ as before, and let $\mathcal{E}=\gamma\left(E^{1}\right)$ be the corresponding continuous choice of orthonormal bases. For each $v \in V$ the intersection $\mathcal{E}_{v}=\mathcal{E} \cap H_{v}$ is an orthonormal basis for the fiber $H_{v}=\ell^{2}\left(s^{-1}(v)\right)$. Since $H_{v}$ is onedimensional, the cardinality of $s^{-1}(v)$ is 1 . Thus the local homeomorphism $s: E^{1} \rightarrow V$ is in fact bijective, and hence is a homeomorphism of $E^{1}$ onto $V$. Thus, replacing the local homeomorphism $s: E^{1} \rightarrow V$ by the isomorphic (in an obvious sense) one id : $V \rightarrow V$, the Hilbert bundle $\mathcal{H}$ is isomorphic to $\mathbb{C} \times V$, and is therefore trivial.

Note that the first part of the above proof did not depend upon the onedimensionality hypothesis.

We can immediately parlay Corollary 6.6 to a result involving correspondences. 
Corollary 6.7. Let $X$ be an A-correspondence whose associated Hilbert bundle $p: \mathcal{H} \rightarrow V$ has one-dimensional fibers. Then $X$ is isomorphic to $X(E)$ for some topological graph $E$ if and only if $\mathcal{H}$ is trivial.

Proof. First assume that $\mathcal{H}$ is trivial. As in the above proof, we can find a continuous choice $\mathcal{E}$ of orthonormal bases for $\mathcal{H}$. We only need to show that for each $v \in V$ the associated representation $\pi_{v}: A \rightarrow B\left(H_{v}\right)$ is diagonalized by the orthonormal basis $\mathcal{E}_{v}=\mathcal{E} \cap H_{v}$. But this is obvious since $H_{v}$ is one-dimensional.

The converse follows immediately from Corollary 6.6.

We will show below in Corollary 6.8 that Corollary 6.7 applies in particular to imprimitivity bimodules over $A$, and then we can make a connection with the Picard group of $A$.

We use the convention that $A$-imprimitivity bimodules are complete ${ }^{1}$, so that such an imprimitivity bimodule $X$ is an $A$-correspondence such that the homomorphism $\phi: A \rightarrow \mathcal{L}(X)$ associated to the left-module action is in fact an isomorphism onto $\mathcal{K}(X)$. By [13, Corollary 3.33], every $A$-imprimitivity bimodule $X$ induces a homeomorphism of $V$, called the Rieffel homeomorphism.

The Picard group of $A$, denoted Pic $A$, is the group of isomorphism classes of $A$-imprimitivity bimodules with group operation balanced tensor product over $A$. The identity element of Pic $A$ is the class of the trivial $A$-imprimitivity bimodule, namely $A$ itself with operations

$$
f \cdot g=f g, \quad\langle f, g\rangle_{A}=\bar{f} g \quad \text { and } \quad{ }_{A}\langle f, g\rangle=f \bar{g}
$$

We call the element of Pic $A$ determined by an $A$-imprimitivity bimodule $X$ the Picard invariant of $X$. By [2, Proposition 3.1] (slightly modified, to turn an antihomomorphism into a homomorphism), the group Aut $A$ of automorphisms embeds as a subgroup of Pic $A$. More precisely, our convention differs from that of [2]: if $\alpha \in$ Aut $A$, we define the associated imprimitivity bimodule of $A$ to be $X=A$ with operations defined for $\xi, \eta \in X$ and $a \in A$ by:

$$
\begin{array}{ll}
- & \xi \cdot a=\xi a ; \\
\text { - } & \langle\xi, \eta\rangle_{A}=\xi^{*} \eta ; \\
\text { - } & a \cdot \xi=\alpha(a) \xi ; \\
\text { - } & { }_{A}\langle\xi, \eta\rangle=\alpha^{-1}\left(\xi \eta^{*}\right) .
\end{array}
$$

Corollary 6.8. Let $X$ be an A-imprimitivity bimodule. Then $X$ is isomorphic to $X(E)$ for some topological graph $E$ if and only if the Picard invariant of $X$ is an automorphism.

Proof. First we show that, because the $A$-correspondence $X$ is in fact an imprimitivity bimodule, it follows that the associated Hilbert bundle $p: \mathcal{H} \rightarrow V$ has one-dimensional fibers. For $v \in V$, recall our notation $J_{v}=\{f \in A: f(v)=0\}$. Let $I_{v}=\overline{\operatorname{span}}\left\{{ }_{A}\left\langle X, X \cdot J_{v}\right\rangle\right\}$ be the ideal of $A$ that is induced by the imprimitivity bimodule $X$. Then by,

\footnotetext{
${ }^{1}$ Unlike in some earlier literature on imprimitivity bimodules between $C^{*}$-algebras, for example $[2,10]$.
} 
for example, [13, Lemma 5.12], the quotient $H_{v}=X /\left(X \cdot J_{v}\right)$ is an $\left(A / I_{v}\right)-\left(A / J_{v}\right)$ imprimitivity bimodule. Since the ideal $J_{v}$ is maximal, so is $I_{v}$, by the properties of the Rieffel correspondence, and so $H_{v}$ is a $\mathbb{C}$-imprimitivity bimodule, and hence, again by the properties of the Rieffel correspondence, is one-dimensional.

Now assume that $X=X(E)$ for a topological graph $E$. By Corollary 6.6 we can assume that the associated Hilbert bundle $p: \mathcal{H} \rightarrow V$ is trivial, and consequently that, as a Hilbert A-module, $X$ is isomorphic to $A$ with operations coming from the multiplication and involution of $A$. It follows that, as an A-imprimitivity bimodule, $X$ is isomorphic to the bimodule associated to an automorphism of $A$.

Conversely, assume that the Picard invariant of $X$ is an automorphism. It follows from the constructions that the associated Hilbert bundle $\mathcal{H}$ is trivial. The result now follows from Corollary 6.7 .

Remark 6.9. Let $X$ be an $A$-imprimitivity bimodule, and let $p: \mathcal{H} \rightarrow V$ be the associated continuous Hilbert bundle. Assume that $X$ is symmetric (borrowing terminology from [1]) in the sense that $a \cdot \xi=\xi \cdot a$ for all $a \in A$ and $\xi \in X$. Since $\mathcal{H}$ has one-dimensional fibers, $\mathcal{H}$ is a complex line bundle (that is, it is locally trivial, by [3, Section 10, Remarque]). Vasselli shows in [16, Proposition 4.3] that, at least when $V$ is compact, the $C^{*}$-algebra $O_{X}$ is commutative, with spectrum the sphere bundle of $\mathcal{H}$.

\section{References}

[1] B. Abadie and R. Exel, 'Hilbert $C^{*}$-bimodules over commutative $C^{*}$-algebras and an isomorphism condition for quantum Heisenberg manifolds', Rev. Math. Phys. 9(4) (1997), 411-423.

[2] L. Brown, P. Green and M. Rieffel, 'Stable isomorphism and strong Morita equivalence of $C^{*}$-algebras', Pacific J. Math. 71 (1977), 349-363.

[3] J. Dixmier and A. Douady, 'Champs continus d'espaces hilbertiens et de $C^{*}$-algèbres', Bull. Soc. Math. France 91 (1963), 227-284.

[4] M. J. Dupré and R. M. Gillette, Banach Bundles, Banach Modules and Automorphisms of $C^{*}$-algebras, Research Notes in Mathematics, 92 (Pitman, Boston).

[5] N. J. Fowler and A. Sims, 'Product systems over right-angled Artin semigroups', Trans. Amer. Math. Soc. 354 (2002), 1487-1509.

[6] S. Kaliszewski, N. Patani and J. Quigg, 'Characterizing graph $C^{*}$-correspondences', Houston J. Math. 38 (2012), 751-759.

[7] A. Kumjian and D. Pask, 'Higher rank graph $C^{*}$-algebras', New York J. Math. 6 (2000), 1-20.

[8] A. Kumjian, D. Pask and A. Sims, 'Homology for higher-rank graphs and twisted $C^{*}$-algebras', J. Funct. Anal. 263 (2012), 1539-1574.

[9] N. Patani, ' $C^{*}$-correspondences and topological dynamical systems associated to generalizations of directed graphs', PhD Thesis, Arizona State University, 2011.

[10] I. Raeburn, 'On the Picard group of a continuous trace $C^{*}$-algebra', Trans. Amer. Math. Soc. 263 (1981), 183-205.

[11] I. Raeburn, Graph Algebras, CBMS Regional Conference Series in Mathematics, 103 (American Mathematical Society, Providence, RI, 2005).

[12] I. Raeburn and A. Sims, 'Product systems of graphs and the Toeplitz algebras of higher-rank graphs', J. Operator Theory 53 (2005), 399-429.

[13] I. Raeburn and D. P. Williams, Morita Equivalence and Continuous-trace C*algebras, Mathematical Surveys and Monographs, 60 (American Mathematical Society, Providence, RI, 1998). 
[14] A. Takahashi, 'A duality between Hilbert modules and fields of Hilbert spaces', Rev. Colombiana Mat. 13(2) (1979), 93-120.

[15] A. Takahashi, 'Hilbert modules and their representation', Rev. Colombiana Mat. 13(1) (1979), $1-38$.

[16] E. Vasselli, 'Continuous fields of $C^{*}$-algebras arising from extensions of tensor $C^{*}$-categories', J. Funct. Anal. 199(1) (2003), 122-152.

S. KALISZEWSKI, School of Mathematical and Statistical Sciences, Arizona State University, Tempe, Arizona 85287, USA

e-mail: kaliszewski@asu.edu

NURA PATANI, School of Mathematical and Statistical Sciences, Arizona State University, Tempe, Arizona 85287, USA

e-mail: nura.patani@asu.edu

JOHN QUIGG, School of Mathematical and Statistical Sciences, Arizona State University, Tempe, Arizona 85287, USA

e-mail: quigg@asu.edu 